\section{Salud bucal costarricense: análisis de la situación de los últimos años}

\section{Costa Rican oral health: analysis of the situation in recent years}

\section{Nota Científica}

Ana Cristina López Torres ${ }^{1, a}$, Gustavo Adolfo Bermúdez Mora ${ }^{2, b, c}$

${ }^{1}$ Práctica privada, San José, Costa Rica

${ }^{2}$ Caja Costarricense de Seguro Social, Dirección de Red Integrada de Prestación de Servicios de Salud, Región Brunca, Costa Rica.

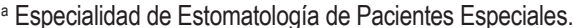

b Supervisor Regional de Odontología.

${ }^{\circ}$ Cirujano Dentista.

\section{Correspondencia:}

Ana Cristina López Torres: cristlopez@gmail.com San Rafael, Montes de Oca, San José, Costa Rica 11504 ORCID: 0000-0001-9074-1897

\section{Coautor:}

Gustavo Adolfo Bermúdez Mora: gabermude@ccss.sa.cr ORCID: 0000-0001-7055-7216

\section{Editora invitada:}

Lita Ortiz-Fernández

Universidad Nacional Mayor de San Marcos, Perú.

Conflicto de intereses: los autores declaran no tener conflicto de intereses.

Fuente de financiamiento: autofinanciado.

\section{Resumen}

Costa Rica ha presentado un progreso en los indicadores de salud bucodental en los últimos 10 ańos, esto gracias a la estructura de su Sistema Nacional de Salud. Los servicios de atención de la salud están divididos en servicio público, privado y otro mixto. La Caja Costarricense de Seguro Social es la entidad que domina el sector público y es el principal, dividido en tres niveles de atención escalonados. Este artículo busca exponer la estructura de las instituciones que conforman la vigilancia y atención de las enfermedades bucodentales más prevalentes en la población costarricense. De la misma manera, presenta el progreso de dichas condiciones a través de los últimos años. Se concluye que, a pesar del progreso obtenido, es necesario el fortalecimiento interinstitucional para afrontar los desafíos en la situación de salud bucodental actual.

Palabras clave: Costa Rica; Salud bucal; Salud pública; Odontología; Políticas públicas (fuente: DeCs BIREME).

\begin{abstract}
Costa Rica has presented progress in oral health indicators in the last 10 years due to its National Health System structure. Health care services are divided into public, private and mixed services. The Costa Rican Social Security Fund is the entity that dominates the public sector and is the main one, divided into three tiered levels of care. This article seeks to expose the structure of the institutions that make up the surveillance and care of the most prevalent oral diseases in the Costa Rican population. In the same way, it presents the progress of these conditions through the last years. It is concluded that, despite the progress made, inter-institutional strengthening is necessary to face the challenges in the current oral health situation.
\end{abstract}

Keywords: Costa Rica; Oral health; Public health; Dentistry; Public policies (source: MeSH NLM).
Recibido: $11 / 06 / 20$

Aceptado: $01 / 07 / 20$

Publicado: 04/08/20 


\section{Introducción}

Costa Rica es un país centroamericano, el cual para el año 2019 poseía una población aproximada de 5058000 habitantes con una esperanza de vida al nacer de 80,2 ańos. Este resultado, es gracias a una estructura sólida en el sistema de salud que viene desarrollándose desde los años 40s ${ }^{1}$.

El Sistema de Salud Costarricense es coordinado por el Ministerio de Salud, que se encarga de la rectoría, la dirección política, la regulación sanitaria, la vigilancia epidemiológica, el direccionamiento de la investigación y el desarrollo tecnológico. Además, está constituido por la Caja Costarricense de Seguro Social (CCSS, única entidad pública a cargo de la prestación de servicios de salud), el Instituto Nacional de Seguros (INS, responsable de la cobertura de los riesgos laborales y de tránsito), el Instituto Costarricense de Acueductos y Alcantarillados (AyA, institución pública rectora y gestora de los servicios de agua potable y saneamiento), los Centros Infantiles de Nutrición y Centros Infantiles de Nutrición y Atención Integral (CEN-CINAI), el Instituto de Alcoholismo y Farmacodependencia (IAFA), el Instituto Costarricense de Investigación y Enseñanza en Nutrición y Salud (INCIENSA), estos tres últimos adscritos al Ministerio de Salud, las universidades, los servicios de salud privados, las municipalidades y las comunidades ${ }^{2}$.

En la Constitución Política de Costa Rica de 1949, se establece el derecho esencial de la salud a partir de los artículos 21 y 50, donde se declara que la vida es inviolable y el Estado debe procurar el mayor bienestar de sus ciudadanos, en un ambiente sano, con protección a la familia como elemento fundamental de la sociedad e igual derecho para "la madre, el niño, el anciano y el enfermo desvalido" 3. La Ley 5395, Ley General de Salud, define de interés público la salud de la población y ordena que el Estado posee entre sus principales funciones brindar prestaciones de salud a todos los costarricenses garantizándoles este derecho, según las leyes y reglamentos ${ }^{4}$. La CCSS, es el principal pilar de la atención de salud en el país; sin embargo, existen servicios de salud privados y mixtos a cargo de empresas de autogestión, aseguradoras, cooperativas, clínicas y hospitales privados ${ }^{5}$.

\section{Salud pública en Costa Rica}

La CCSS creada en 1941 mediante la Ley No. 17, es la institución encargada de administrar los seguros sociales bajo los principios de universalidad, solidaridad y equidad. Proporciona los servicios de forma integral, al individuo, la familia y la comunidad, otorga protección social, económica y de pensiones a los costarricenses según la legislación vigente. Se encuentra conformada por un conjunto de establecimientos de salud bien organizados e interrelacionados entre sí, operando bajo un modelo de redes, el cual contribuye al aseguramiento de la continua prestación de servicios, divididos por niveles de atención (capacidad resolutiva y manejo según grados de complejidad) en siete regiones con un total de 106 áreas de salud, 1064 centros de salud compuestos por Equipos Básicos de Atención Integral en Salud
(EBAIS) y 29 hospitales ( 3 nacionales, 6 especializados, 8 regionales y 12 periféricos). Esta red busca satisfacer las necesidades y demandas de la población y acrecentar la capacidad operativa de la institución ${ }^{6,7}$.

El seguro social se creó para la atención de la población trabajadora y este se extendió a nuevos beneficiarios estrechamente ligados a la categoría de contribuyentes y no contribuyentes. Existen diferentes clasificaciones para acceder a este seguro: 1. Asegurados directos (trabajadores asalariados, pensionados, asegurados directamente por el Estado, como lo es la población en condiciones de pobreza, menores de 18 años, mujeres embarazadas sin protección por un familiar beneficiario e indigentes identificados), 2. Asegurados indirectos (familiares y dependientes de asegurados directos) y 3. No asegurados (personas con capacidad contributiva que no aportan a la seguridad social y migrantes indocumentados) ${ }^{2,5}$.

El financiamiento de esta institución se hace por medio de una cotización tripartita en la que se encuentran los empleadores o patrones, trabajadores y el Estado. Al poner de ejemplo, el salario mínimo mensual estipulado en el presente año por el Ministerio de Trabajo y Seguridad Social, que ronda los $\mathbb{2} 200000$ - colones (unos $\$ 350$ USD), la CCSS recibe un aporte salarial correspondiente al Seguro de Enfermedad y Maternidad (SEM) y al Seguro de Invalidez Vejez y Muerte (IVM), el cual representa actualmente para el trabajador un 9,5\% y para el patrono un $14,5 \%$ del salario de su empleado, lo que respectivamente serían $\$ 19000$ (unos \$33 USD) y \$29000 (unos $\$ 51$ USD) según el ejemplo dado. Por su parte, el Estado aporta en total 0,66\% del salario de todos los trabajadores asalariados del país ${ }^{5,8-10}$.

El Estado dispone del Régimen no Contributivo, el cual, por medio de la CCSS, brinda la atención a la población en condiciones de pobreza, permitiendo el acceso a la salud por igual en la población, sin importar su condición económica.

Con la abolición del ejército en 1948, el país ha conseguido destinar un mayor porcentaje del Producto Interno Bruto (PIB) para la salud y educación de la población. Según datos de la Comisión Económica para América Latina y el Caribe (CEPAL), en 1950 el país realizó un gasto en salud correspondiente al 2,2\% del PIB, el cual a través de las décadas ha ido en aumento. En el año 2016 la inversión en salud alcanzó el 7,8\%, cumpliendo con el 6\% establecido por la Organización Panamericana de la Salud (OPS) a través de la Estrategia para el Acceso Universal a Salud y Cobertura Universal de Salud, propuesto para los países miembros de la OPS en el $2014^{2,11,12}$.

\section{Sistema de salud bucal en Costa Rica}

Costa Rica cuenta con cuatro universidades que brindan la carrera de Licenciatura en odontología, una estatal y tres privadas. Para el 2018 se reportó que existían 9,7 odontólogos por cada 10000 habitantes ${ }^{13}$. Según el Colegio de Cirujanos Dentistas del país (CCDCR), Costa Rica actualmente cuenta con 5190 odontólogos activos 
en práctica pública y privada, entre ellos 977 odontólogos especializados en 17 especialidades diferentes ${ }^{14}$.

En la CCSS se brinda el servicio de odontología de manera gratuita a todas las personas aseguradas. Este cuenta con tres diferentes niveles de atención, en los cuales se accede de manera escalonada hacia los niveles de más alta complejidad ${ }^{15,16}$ :

1. Primer nivel: el programa trabaja bajo el concepto de Atención Integral de la Salud y forma parte de los EBAIS, es el nivel con mayor grado de cobertura, siendo del $75 \%$ del recurso humano profesional. Se encarga de la promoción de la salud con participación social, prevención primaria individual y colectiva, atención de alteraciones incipientes de salud bucodental. Además, realiza la vigilancia de la condición de la salud bucodental a todo el sistema, esta información es registrada de manera diaria a lo largo del año: los estados de las piezas dentales, estado de condición periodontal, planos cráneo- faciales y apiñamiento dental; con esta información se evalúa el impacto en la población de los servicios prestados. Para este nivel se ejecutan tratamientos de baja y media complejidad como son: prevención, profilaxis dental, operatoria dental, periodoncia, programas de Guía de la Erupción Dental (PROGEO), exodoncias simples y de compromiso parcial de tejidos blandos, endodoncias unirradiculares y referencias en casos necesarios. Posee diferentes modalidades para operar, como lo son los servicios fijos, transportables y móviles.

2. Segundo nivel: corresponde al 15\% de los recursos humanos profesionales. Este nivel está relacionado a la especialidad básica de Odontología General Avanzada (OGA) y algunas otras, las cuales brindan atenciones de mayor complejidad y a pacientes con condiciones de discapacidad y sistémicas de atención especial, tanto de tipo ambulatorio como hospitalario. Este equipo profesional opera según la evidencia epidemiológica y demográfica, suministrada por el nivel base de la red. Su función corresponde a la resolución de casos de mayor complejidad según orden de prioridades y trabajo en conjunto con el primer nivel. Su actuar está relacionado a periodoncia, endodoncia, exodoncias complejas, cirugía bucodental menor, ortopedia funcional, dolor miofacial, trastornos de la articulación temporomandibular, odontopediatría y tratamientos protésicos en el adulto mayor. Entre otras funciones de este nivel se encuentra la promoción y prevención de la salud y capacitación al personal del primer nivel.

3. Tercer nivel: corresponde al $10 \%$ de recurso humano profesional, el cual se encuentra en Hospitales Nacionales Generales y Especializados. Cumple acciones de atención clínica correctiva de alteraciones y enfermedades de la salud bucodental. Su actuar puede ser de tipo ambulatorio, así como hospitalario. Las prestaciones son brindadas por odontólogos especialistas que realizan la atención clínica de condiciones de la más alta complejidad.
Para el año 2017, se contabilizaron en total 2315312 consultas en los centros de atención odontológica de la CCSS, cantidad que se mantiene relativamente constante desde el año $2010{ }^{17}$.

También desde 1951, se crearon los Centros de Educación y Nutrición y de Centros Infantiles de Atención Integral (CEN-CINAI), tras haber identificado la desnutrición infantil como un problema de salud. Estos se encargan, en conjunto con el Ministerio de Salud, de brindar alimentación a la población menor de 13 años, a mujeres embarazadas o en periodo de lactancia en condición de pobreza extrema o riesgo social. Dentro de los servicios que brinda la institución, se encuentra los controles de índice de placa bacteriana, los cuales realiza dos o tres revisiones anuales. Además, proporciona cepillos, pastas dentales y programas de educación en salud bucodental a los niños y adultos. Los casos que requieren de atención bucodental son referidos a los establecimientos de salud de la CCSS que les corresponde ${ }^{18,19}$.

\section{Situación epidemiológica bucal del país}

Según Brenes, en el último estudio realizado en el país que evalúa el CPOD, en la franja de los 12 años ha tenido una tendencia a disminuir entre los años 1966 al 2006. Durante estos años, se observa una disminución de 9,23 en el CPOD de 1966 hasta un 2,57 en el 2006 en el estudio de Montero et al. Este mismo revela que los componentes del índice CPOD del 2006 se distribuyen de la siguiente manera: un 55\% a piezas obturadas, $39 \%$ a piezas con caries dental activa y un $5,4 \%$ a piezas dentales perdidas por caries dental ${ }^{20,21}$.

En el caso de los CEN-CINAI, la Dirección Nacional de dicho programa establece la programación de evaluaciones de higiene oral de los niños menores de 7 años. Para el año 2009 se realizaron en dos momentos del año, participando 15086 niños en la primera y 15411 en la segunda. Los resultados fueron poco alentadores, ya que en la primera evaluación, la mala higiene oral encabezó los porcentajes con 59,56\% seguido de la higiene regular con 17\%; posteriormente, en la segunda evaluación se registró $54,56 \%$ en la condición de mala higiene y $17,40 \%$ en la de higiene regular. Entre las metas propuestas se encontraba el fortalecer las estrategias de mejora de higiene oral que permitieran disminuir los problemas de dentición primaria ${ }^{22}$. En el 2015, al realizarse las mismas evaluaciones se encontró una mejoría; en la primera evaluación con 15693 niños, el porcentaje de higiene óptima y buena fue de $46,5 \%$ y de higiene regular y mala del $53,4 \%$. En la segunda evaluación de dicho año, los 15088 niños obtuvieron porcentajes de 52,7\% y $47,3 \%$, respectivamente. Las estrategias utilizadas presentan un cambio en el porcentaje a favor de la buena salud oral pero el porcentaje de higiene mala y regular sigue siendo casi del $50 \%$ de la población ${ }^{23}$. En el último informe del 2018, se realizaron tres evaluaciones, donde se contabilizaron 13355 niños en la primera, 12726 en la segunda y 11477 para la última. Los resultados para la higiene bucal óptima y buena fueron 45,8\%, 51,2\% y $55,6 \%$ respectivamente. Se puede observar que en los 
años comparados existe una diferencia a favor de una mejor higiene oral entre la primera y segunda/tercera evaluación, pese a esto, sigue observándose un porcentaje elevado de menores con higiene regular y mala ${ }^{18}$.

En el 2014, el Sistema de Vigilancia Epidemiológica en Odontología (SIVEO), perteneciente a CCSS, evaluó la salud dental en 80071 asegurados, donde consideraron las piezas sanas, perdidas y cariadas, en diferentes grupos de edad, los cuales fueron de 0 a 5, 6, 7 a 9, 10 a 12, 13 a 19, 20 a 45, 46 a 59, 60 y más ańos. En el estudio se observó que la población posee un porcentaje elevado de piezas sanas, de los 0 hasta los 19 años. Posterior a esto, existe una disminución acelerada de la condición de dichas piezas en asegurados de 20 hasta 60 años o más, con un aumento de la pérdida dental, llegando hasta el $77 \%$ en la población adulta mayor. La caries dental presenta una continua disminución en todas las etapas de la vida, pero inicia su reducción más relevante desde los 13 a 19 años hasta la población de 60 y más años, simultáneo a la pérdida dental abrupta (Figura 1) ${ }^{24}$.

Otro estudio del SIVEO en el $2016^{25}$, con 504086 adultos mayores de 60 años que asistieron a los Servicios de Odontología de la CCSS (SOCCSS) durante el año, encontró que la prevalencia de periodonto sano fue únicamente de $11,89 \%$, en cambio, los datos de enfermedad periodontal registraron un $24,11 \%$ y el $63,99 \%$ se encontraba edéntula debido a diferentes alteraciones periodontales. La enfermedad periodontal se subdividió según el hallazgo de: sangrado gingival en el 5,83\%, cálculo dental en $13,55 \%$, bolsas periodontales de 4-5 $\mathrm{mm}$ en 3,43\% y bolsas de más de $5 \mathrm{~mm}$ en $1,29 \%$. La media de dientes naturales presentes fue de 5 , mientras que en los ausentes fue de 21. El análisis de la condición de piezas dentales reveló que el $5,4 \%$ presentaban una corona sana, $6,6 \%$ corona obturada, $7,2 \%$ coronas con caries, $21,72 \%$ pérdida dental por caries y un $58,64 \%$ pérdida dental por otro motivo. Entre otros datos relevantes, el 13,55\% de la población presentó algún trastorno en la articulación temporomandibular. En cuanto al uso de prótesis dentales, el $54 \%$ poseía algún tipo; siendo la prótesis total removible la más prevalente con un $36,65 \%$ en el caso de las maxilares y la prótesis parcial removible en un $22,10 \%$ de las mandibulares.

En el 2017, se realizó un estudio en 911830 adolescentes en rangos de edad de 10 a 19 años, que fueron atendidos en los SOCCSS. Se reveló que el 13,14\% de la población estudiada tenía afecciones del esmalte causadas por el aumento de fluoruros. También menciona que las piezas dentales se encontraban sanas en $82 \%$, el resto se encontraba dividido en un $4 \%$ con piezas con sellantes dentales, $6,33 \%$ con piezas obturadas con resina o amalgama, $6,77 \%$ caries en la corona, $0,19 \%$ con pérdida de la corona por caries y un $0,87 \%$ con pérdida de la corona por otro motivo. Además, se evidenció una prevalencia de apińamiento dental de algún tipo en $32,69 \%$ de la población, siendo $19,71 \%$ en un único segmento y $12,98 \%$ en dos segmentos ${ }^{26}$.

Lao y Araya ${ }^{27}$, evaluaron la prevalencia de enfermedad periodontal en Costa Rica durante el año 2017 por medio de un estudio transversal, con 105075 personas, con información recopilada en los diferentes SOCCSS y empleando el Índice de las Necesidades Terapéuticas Periodontales de la Comunidad (INTCP) desarrollado por la Organización Mundial de la Salud (OMS) y empleado como gold standard en estudios epidemiológicos de esta condición. Se encontró que la prevalencia de la condición higiénica fue $58,55 \%$, la enfermedad periodontal $35,36 \%$ y la pérdida de piezas dentales por enfermedad periodontal $6,36 \%$.

El factor higiénico (correspondiente a una adecuada condición de higiene oral) evaluado por grupos de edad reveló que esta condición presenta una prevalencia del

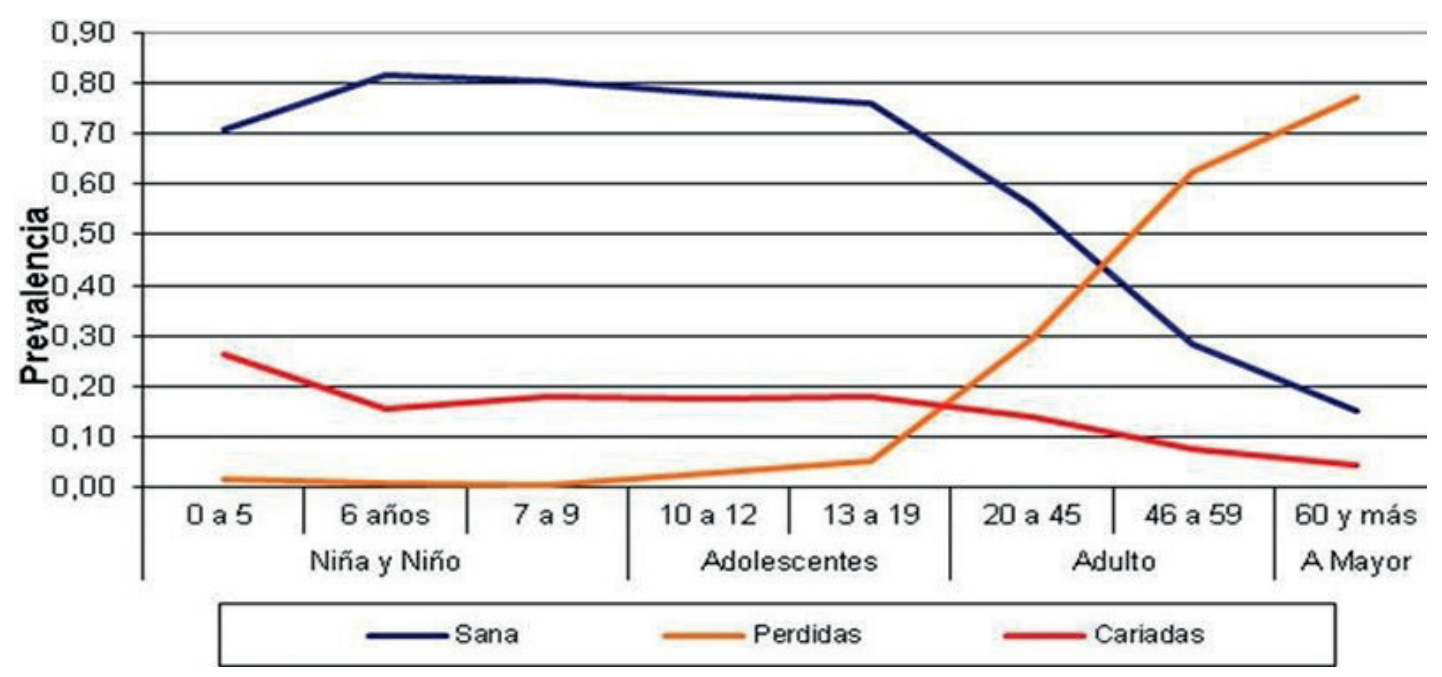

Figura 1. Comparación de la prevalencia de la condición dental por grupos de edad, CCSS, Costa Rica, SIVEO, 2014 Fuente: SIVEO, CCSS, 2014. 
$93,23 \%$ en la población de $0-5$ años y se mantiene con valores ligeramente menores de hasta el $82,38 \%$ en el grupo de 10 a 12 años. A partir de esta edad, se observó una disminución progresiva de la condición conforme incrementa la edad, hasta llegar al grupo de mayores de 60 ańos con un 21,34\%. En el caso de la enfermedad periodontal, se reportó un 6,29\% de los 0-5 ańos y un incremento durante la vida hasta llegar a 59,10\% en las edades de 20-45 años y posteriormente a 40,64\% en la población adulta mayor, relacionado con el incremento de la pérdida de piezas dentales. El sextante nulo mostró valores bajos de hasta 2,60\% en los grupos menores de 20 a 45 ańos, posteriormente presentó un cambio drástico en edades mayores con un $37,83 \%$ en el adulto mayor ${ }^{27}$.

En el 2019, la Coordinación Nacional de Odontología de la CCSS presentó el informe "Situación de la Salud Oral en Costa Rica durante los años 2009 a 2018” ${ }^{28}$, en donde describió las condiciones orales más prevalentes y las variables epidemiológicas de tiempo, lugar y persona. Se utilizaron gráficos de las frecuencias de salud/ enfermedad, conocidos como "corredores o canales endémicos" que permitieron describir las condiciones de salud bucal en un periodo de tiempo (datos del SIVEO del 2009 al 2017) y se compararon con el comportamiento observado en el ańo 2018. Los indicadores compuestos en odontología revelaron que desde el punto de vista bucodental existe una mejoría a través de los años comparados (canal endémico) con los datos del SIVEO 2018. Este documento menciona:

- Las condiciones de pieza sana y factor higiénico mejoraron en todos los grupos de edad con excepción del grupo de 6 años.

- El riesgo bucodental (caries, enfermedad periodontal, problemas de planos craneofaciales y apińamiento dental) se mantuvo en el límite inferior del canal endémico para la población de 0 a 6 años y en el grupo de mayores de 60 años; mientras que los otros grupos poblacionales presentaron una reducción de dicho riesgo.

- La pérdida dental, por causas de enfermedad periodontal o caries, se mantuvo igual en la población de los 0 hasta los 19 años, pero se observó la disminución del indicador en los otros grupos de edad.

- En la condición de pieza dental sana, los grupos presentaron una mejoría con excepción del grupo de 20 a 45 ańos que mantuvo la condición de los ańos anteriores (Figura 2).

- En cuanto a la pieza dental cariada, se presentó una reducción importante en la población de 0 hasta los 35 ańos en mujeres, continuó constante en el grupo de 20 a 45 ańos, pero empeoró a partir del grupo de 46 años y más (Figura 3).

- La enfermedad periodontal presentó una disminución en los grupos de 0 hasta los 19 años, se mantuvo constante en la mujer de 20 a 35 años. Sin embargo, exhibió un aumento en todos los grupos de más de 36 años.

- El sextante nulo presentó una mejoría en los grupos de 0 a 6 años, en mujeres de 20 hasta los 45 años y en la población de ambos sexos de los 46 a más años. No obstante, en las edades de 7 a 19 años y en hombres de 20 a 45 años se mantuvo igual a los corredores endémicos.

\section{Políticas y estrategias}

El Plan Nacional de la Salud 2016 a $2020^{29}$, es el instrumento con el cual se operacionaliza la Política Nacional de Salud "Dr. Juan Guillermo Ortiz Guier”. Estas políticas poseen como orientadores principalmente a la equidad, calidad e inclusividad social. El fortalecimiento del

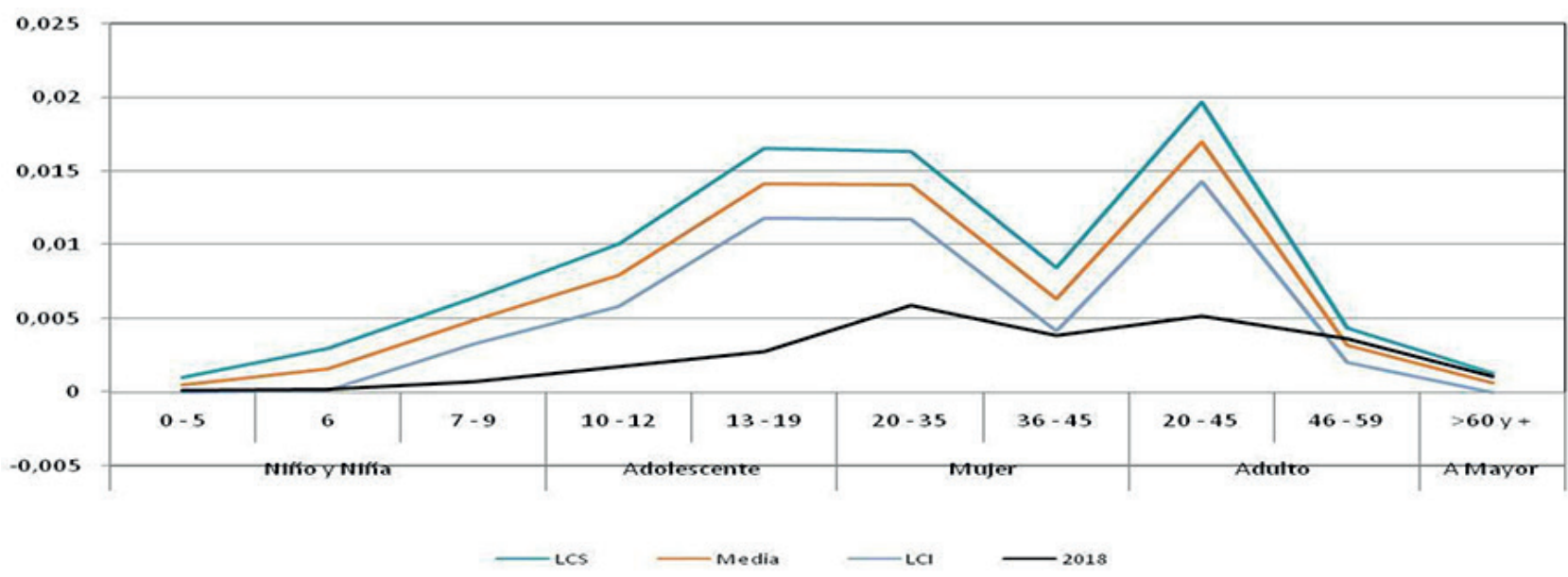

Figura 2. Curva epidemiológica por grupos de edad. Índice de Riesgo Bucodental 2018 en comparación con gráficos de control 2009 a 2017, SIVEO, CCSS, Costa Rica

Fuente: SIVEO 2008 al 2017, CCSS, Coordinación Nacional de Odontología, 2018.

Documento: CCSS, Situación de la Salud Oral en Costa Rica durante los años 2009 a 2018. 


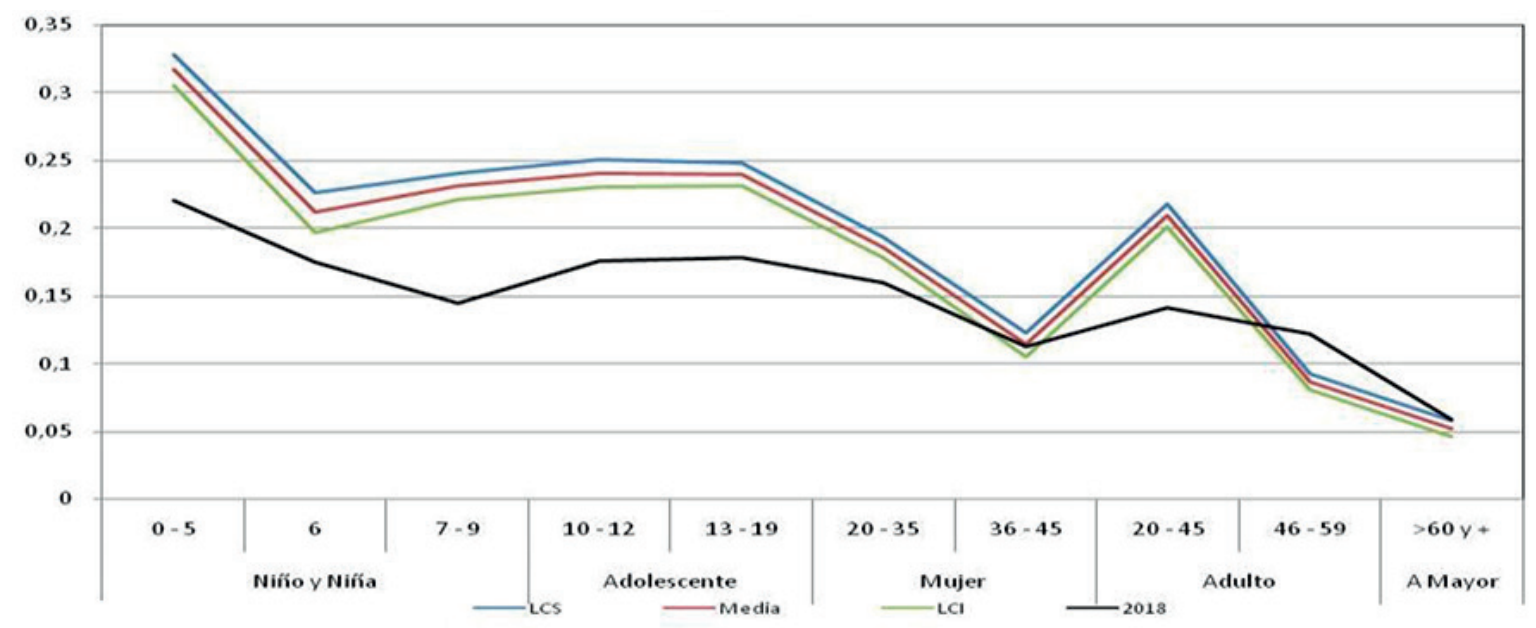

Figura 3. Curva epidemiológica por grupos de edad. Pieza cariada 2018, en comparación con gráficos de control 2009 a 2017, SIVEO, CCSS, Costa Rica

Fuente: SIVEO 2008 al 2017, CCSS, Coordinación Nacional de Odontología, 2018

Documento: CCSS, Situación de la Salud Oral en Costa Rica durante los años 2009 a 2018.

sistema de salud desde la perspectiva de los determinantes sociales de la salud fue una de las políticas destacadas en el 2015, contando con estrategias como el desarrollo de modelos de atención integral, a partir del fortalecimiento de la estrategia de atención primaria renovada en salud (APS).

En el Área de Intervención de Atención Primaria de Salud Bucodental se ha buscado fortalecer este eje en la población por medio de dos programas o iniciativas específicas con apoyo del CCDCR. El primero fue a través del "Programa 60 y más", con el objetivo de promover la salud bucodental para un envejecimiento activo en la población adulta mayor de la Red Nacional de Clubes de la Asociación Gerontológica Costarricense (AGE$\mathrm{CO})$ por medio de talleres educativos y revisiones odontológicas. La segunda iniciativa ha sido impulsada con miras a diseñar una Política de Protección de la Salud Bucodental, que permita desarrollar un plan de promoción, prevención y atención que articule los esfuerzos interinstitucionales y se fomenten y operacionalicen propuestas conjuntas para atender las necesidades de la población, en especial de los grupos más vulnerables ${ }^{30}$.

En el documento sobre la "Situación de la Salud Oral en Costa Rica durante los años 2009 a 2018" de la CCSS, se mencionan algunas estrategias institucionales que deben llevarse a cabo para buscar el mejoramiento de la salud bucodental, como lo son: las evaluaciones de factores de riesgo y determinantes de la salud de la población de cada región del país, la coordinación con otras instituciones (como centros educativos, gobiernos locales, Juntas de Salud, entre otros) para el desarrollo de programas de educación popular que permitan interiorizar las condiciones de riesgo y busquen realizar los cambios necesarios hacia estilos de vida saludables. Además, se destaca la necesidad de promover el desarrollo de programas interdisciplinarios para la atención integral de la población durante el curso de la vida, fundamentados en principios de equidad, calidad y efectividad ${ }^{28}$.

\section{Retos futuros}

Se hace necesaria la elaboración de una política pública en el país que oriente y defina objetivos, estrategias, presupuestos y entidades responsables de las diferentes líneas de promoción, prevención y atención de la salud bucodental de la población. Esta misión debe ser encabezada por el Ministerio de Salud, trabajando desde las áreas públicas y privadas, en trabajos interdisciplinarios, que incluyan centros de salud, universidades, escuelas y colegios, gobiernos locales, entre otras entidades que son parte del sector salud o tienen relación directa/indirecta. El enfoque en los determinantes sociales de la salud (considerando aspectos como los hábitos y estilos de vida, entornos ambientales, factores biológicos individuales, acceso a los sistemas sanitarios, entre otros), debe ser transversal en las propuestas de acción y articulación interinstitucional para lograr mayor impacto y mejores resultados en beneficio de los individuos y sus familias.

La currícula de las universidades que imparten la odontología debe dar énfasis a la formación en la salud bucal comunitaria, que permita a los profesionales desarrollarse e identificar en la población las principales necesidades y determinantes de la salud. Esto, con el fin de fomentar la participación social, permitiendo trabajar más en esfuerzos dirigidos a la promoción de la salud y la prevención primaria de las enfermedades bucodentales con alta prevalencia.

Se requiere fortalecer las iniciativas en promoción de la salud y prevención de la enfermedad, tanto a nivel bucal como sistémico, a través de la educación y el involucramiento colectivo que permita generar agentes multiplicadores en las comunidades, con el fin de mejorar los estilos de vida de las personas y disminuir el riesgo de afecciones causadas por factores modificables relacionados a dietas poco saludables, particularmente aquellas con alto contenido de azúcar, además del consumo de tabaco y bebidas alcohólicas. 
Es importante abordar integralmente y evaluar la asociación entre las enfermedades crónicas no transmisibles (cáncer, diabetes, obesidad, enfermedades cardiovasculares, respiratorias crónicas, entre otras) y las enfermedades bucodentales que presentan los costarricenses, ya que son altamente prevalentes en la población y con el aumento de esperanza de vida, se incrementa el riesgo de sufrir estos padecimientos. Como ejemplo, en el 2014 la CCSS menciona que la diabetes mellitus se encontró diagnosticada en aproximadamente el $10 \%$ de la población y la hipertensión arterial en el $31,2 \%$, ambas condiciones poseen un efecto sobre la condición de salud oral haciendo más susceptibles a quienes las padecen a presentar caries y enfermedad periodontal ${ }^{31}$.

Según datos del Instrumento de Gestión de los Servicios Odontológicos (IGEO) de la CCSS ${ }^{33}$, en el 2018, dentro de la institución, se contabilizaron 540 asistentes dentales y 604 odontólogos, de los cuales 442 eran generales, 128 especialistas y el resto ocupaba puestos administrativos y gerenciales. La relación odontólogo-población institucional fue de 1 odontólogo general por cada 11570 habitantes en el primer nivel de atención. Esto dista de la cantidad estipulada según el Plan Nacional de Atención en Salud Bucodental de la CCSS ${ }^{15}$, el cual menciona que la relación debería ser de 1 odontólogo por cada 5000 personas, al mismo tiempo, la OMS ${ }^{32}$ recomienda que esta proporción sea de un profesional por cada 3500 habitantes. La necesidad de una mayor contratación de profesionales del área de odontología es evidente e indispensable para poder brindar mayor atención a la población, identificar las enfermedades en sus estadios iniciales y desarrollar proyectos educativos enfocados en la protección y promoción de la salud oral.

Así mismo, el IGEO 2018 reportó una cobertura de los SOCCSS en $17,8 \%$ de la población asegurada, siendo mayor el alcance en los grupos de niños y adolescentes, y mucho menor en el adulto mayor de 65 años. Esto evidencia una brecha en el acceso de los programas de salud bucal, ya que, a pesar de los esfuerzos por alcanzar a la mayor cantidad de personas, la capacidad actual del sistema se ve limitada por los recursos institucionales disponibles que no son suficientes ante la transición demográfica y situación epidemiológica que vive el país ${ }^{33}$.

En el 2015, los adultos mayores de 65 años representaron el $7 \%$ de la población costarricense; sin embargo, con base en proyecciones del Instituto Nacional de Estadística y Censo (INEC), para el año 2040, se espera que esa cifra aumente a $18 \%$, pudiendo llegar a contabilizarse más de un millón de personas para ese año. Esto, significa que la población cotizante irá decreciendo y simultáneamente la población adulta mayor será más numerosa, lo que requerirá de nuevas estrategias de ingreso y financiamiento para atender las necesidades de la población. El campo de la odontología no será la excepción, ya que deberá responder a las demandas de prestaciones específicas para estos grupos mediante una mejor y mayor oferta de servicios tales como prótesis dentales y procedimientos rehabilitadores y de mantenimiento, además de tratamientos especializados en odontogeriatría.

\section{Conclusiones}

El modelo solidario, universal y obligatorio del seguro social de salud en Costa Rica a cargo de la CCSS ha permitido la integración de los servicios odontológicos dentro del paquete de prestaciones de servicios públicos en beneficio de la población asegurada del país.

En general, Costa Rica ha presentado una mejora en sus indicadores de salud bucodental, como se muestra al comparar el CPOD de 1966 al 2006 y la disminución de prevalencia de enfermedades bucodentales con los datos de la CCSS del canal endémico y el año 2018. Sin embargo, existen múltiples retos para el perfeccionamiento de la situación de salud oral del país. Algunas de las condiciones evaluadas se encuentran en la parte inferior de los canales endémicos, es decir, se mantienen constantes y no se observa una disminución importante en sus cifras. La población adulta y adulta mayor siguen viéndose afectados por una mayor prevalencia de caries y enfermedad periodontal, por lo que deben impulsarse y fortalecerse los programas de atención y prevención primaria en salud oral, con el fin de evitar el progreso a condiciones más severas que lleven a la pérdida dental.

Es importante que se desarrolle un plan de trabajo, a través de políticas concretas e integrales, entre las distintas instituciones que velan por el bienestar de la población en general, no solo a través de las pertenecientes al sector salud, sino también con el apoyo de otras como el Ministerio de Educación, Ministerio de Ambiente, colegios profesionales, universidades, organizaciones de la sociedad civil y otras encargadas de brindar apoyo y atención a poblaciones en riesgo (adultos mayores, indígenas, personas con discapacidad, indigentes y grupos urbano-marginales, entre otros.). Esto, con el fin de generar alianzas y articulaciones inter- y multidisciplinarias que permitan contrarrestar las inequidades sociales de la salud, tanto a nivel bucodental como general, en especial, de los grupos más vulnerables de la sociedad.

La necesidad de incrementar la cantidad de personal profesional en la atención primaria de los servicios en odontología es fundamental para responder a la evidente desproporcionalidad que existe entre cantidad de odontólogos versus población de un área. Esto permitiría distribuir mejor las labores enfocadas en proyectos educativos según grupos etarios, aumentar las prestaciones por región geográfica y cubrir más escenarios de trabajo según análisis epidemiológico, reforzando el abordaje temprano en escuelas, colegios, centros de cuido (guarderías y hogares de ancianos) y creando nuevas estrategias o reforzando programas de EBAIS, comunidades lejanas, entre otros, para asegurar el acceso equitativo a todas las personas que habitan el territorio costarricense. 


\section{Referencias bibliográficas}

1. Instituto Nacional de Estadístico y Censos. Estadísticas demográficas 2019 [Internet]. 2020 [citado 12 de mayo de 2020]. Disponible en: https://www.inec.cr/poblacion/temas-especiales-de-poblacion

2. Ministerio de Salud. Informe del Sistema de Cuentas de Salud de Costa Rica, 2011-2016 [Internet]. San José, Costa Rica; 2018 [citado 25 de mayo de 2020] p. 169. Disponible en: https://www.ministeriodesalud.go.cr/index.php/biblioteca-de-archivos/ centro-de-informacion/material-publicado/publicaciones-guia-metodologica-para-la-aplicacion-del-sistema-de-cuentas-de-salud-2011/4030-informe-del-sistema-de-cuentas-salud-cr-2011-2016/file

3. República de Costa Rica. Constitución Política de Costa Rica. San José Costa Rica: Asamblea Legislativa; 1949.

4. República de Costa Rica. Ley General de la Salud. Ley 5395. Sec. Art. 3, San José, Costa Rica Asamblea Legislativa, 1973.

5. Sáenz M, Acosta M, Muiser M, Bermúdes J. Sistema de salud de Costa Rica. Salud Pública Méx. 2011;53 suppl 2:S156-S167.

6. Dirección de Gestión de Calidad Ambiental. DIDECA. Estado de Situación: Uso de Amalgama y minería artesanal de oro en Costa Rica [Internet]. Estado de situación: Uso de Amalgamas y Minería Artesanal de oro en Costa Rica. [citado 10 de mayo de 2020]. Disponible en: http://www.digeca.go.cr/sites/default/files/documentos/2_estado_situacion_uso_d e_amalgamas_y_mineria_artesanal_de_oro_en_costa_rica_0.pdf

7. Caja Costarricense de Seguro Social. Memoria Institucional 2018 [Internet]. San José, Costa Rica: Caja Costarricense del Seguro Social; 2019 [citado 10 de mayo de 2020] p. 253. Disponible en: https://repositorio.binasss. sa.cr/repositorio/handle/20.500.11764/720

8. Caja Costarricense de Seguro Social. Calculadora Patronal [Internet]. Calculadora Patronal. 2020 [citado $26 \mathrm{de}$ mayo de 2020]. Disponible en: https://www.ccss.sa.cr/ calculadora

9. Caja Costarricense de Seguro Social. Reglamento del Seguro de Salud [Internet]. 2014 p. 36. Disponible en: https://www.ccss.sa.cr/normativa?pagina $=2 \&$ cat $=18$

10. Caja Costarricense de Seguro Social. Reglamento del Seguro de Invalidez Vejez y Muerte [Internet]. Art. 33. Disponible en: https:/www.ccss.sa.cr/normativa?pagina $=2 \&$ cat $=18$

11. Cid C, Matus M, Báscolo E. Espacio fiscal para salud en las Américas: ¿̇es suficiente el crecimiento económico? Rev Panam Salud Pública. 2018;42:e86.

12. Güendel L, Trejos J. Reformas recientes en el sector Salud de Costa Rica [Internet]. Santiago, Chile: Comisión Económica para América Latina y el Caribe; 1992 [citado 1 de junio de 2020] p. 52. Disponible en: https:// repositorio.cepal.org/bitstream/handle/11362/33814/ S9200608_es.pdf

13. Organización Panamericana de la Salud 2019. Indicadores básicos 2019: Tendencias de la salud en las Américas. Washington, DC; 2019.
14. Colegio de Cirujanos Dentistas de Costa Rica. Miembros activos [Internet]. [citado 12 de mayo de 2020]. Disponible en: http://www.colegiodentistas.org/sitCol/ miembros-activos/

15. Caja Costarricense de Seguro Social. Plan Nacional de Atención en Salud Bucodental 2004. San José, Costa Rica; 2004.

16. Caja Costarricense de Seguro Social. Norma de Prestaciones Odontológicas, junio 1997 [Internet]. San José Costa Rica; 1997 [citado 15 de mayo de 2020]. Disponible en: https://www.ccss.sa.cr/publicaciones

17. Caja Costarricense de Seguro Social. Estadísticas de Salud. Anuarios Estadísticos del 2010 - 2017 [Internet]. 2020 [citado 27 de mayo de 2020]. Disponible en: https://www.ccss.sa.cr/est_salud

18. Dirección Nacional de CEN-CINAI. Informe Datos Básicos: perfil socioeconómico de los beneficiarios y vigilancia de la salud, crecimiento y desarrollo infantil 2018 [Internet]. San José, Costa Rica: Ministerio de Salud; 2020 [citado 24 de mayo de 2020] p. 97. Disponible en: https://www.cen-cinai.go.cr/images/pdf/Informes/Informe_Datos_Bsicos2018_.pdf

19. Dirección Nacional de CEN-CINAI. Tipos de establecimiento [Internet]. 2018 [citado 3 de junio de 2020]. Disponible en: https://www.cen-cinai.go.cr/index.php/ cen-cinai/tipo

20. Brenes W. Epidemiología bucal, respuesta social y desafíos para el desarrollo de comunidades promotoras de salud en Costa Rica. En: La Salud Pública en Costa Rica, estado actual, retos y perspectivas. Escuela de Salud Pública, 1 edición. San José, Costa Rica: Editorial Universidad de Costa Rica; 2011.

21. Montero O, Ulate J, Rodríguez A, Méndez C, Elías A. Prevalencia de caries dental en niños y niñas escolares de 12 años de edad en Costa Rica, 2006. Rev Cient Odonto. $2011 ; 7(2): 55-63$.

22. Dirección Nacional de CEN-CINAI. Informe Datos Básicos 2009 [Internet]. San José, Costa Rica: Ministerio de Salud; 2010 [citado 24 de mayo de 2020] p. 28. Disponible en: https://www.cen-cinai.go.cr/images/pdf/ Informe\%20Datos\%20Bsicos\%202009.pdf

23. Dirección Nacional de CEN-CINAI. Informe Datos Básicos 2015 [Internet]. San José, Costa Rica: Ministerio de Salud; 2017 [citado 24 de mayo de 2020] p. 94. Disponible en: https://www.cen-cinai.go.cr/images/pdf/ Informe $\% 20$ Datos $\% 20$ Bsicos $\% 202015$.pdf

24. Caja Costarricense de Seguro Social. Epidemiología en odontología [Internet]. Vigilancia epidemiológica. sf [citado 18 de mayo de 2020]. Disponible en: https://www. ccss.sa.cr/odontologia?epidem

25. Lao W. Estudio epidemiológico de la Salud Oral del Adulto Mayor en Costa Rica, 2016. San José Costa Rica: Caja Costarricense de Seguro Social; 2017 p. 30.

26. Lao W. Estudio epidemiológico de la Salud Oral del Adolescente en Costa Rica, 2017. San José, Costa Rica: Caja Costarricense del Seguro Social; 2018 p. 30.

27. Lao W, Araya H. Enfermedad periodontal en Costa Rica 2017. Odontol VITAL. 2018;2(29):59-68. 
28. Caja Costarricense de Seguro Social. Situación de la Salud Oral en Costa Rica durante los ańos 2009 a 2018. San José, Costa Rica: CCSS; 2019 p. 46. Report No.: DRSS-0087-18.

29. Ministerio de Salud. Política Nacional de Salud «Dr. Juan Guillermo Ortiz Guier». San José Costa Rica; 2015 p. 154.

30. Ministerio de Salud. Plan Nacional de Salud 2016-2020 [Internet]. San José, Costa Rica; 2016 [citado 15 de mayo de 2020] p. 212. Disponible en: https://www.ministeriodesalud.go.cr/index.php/biblioteca-de-archivos/sobre-el-ministerio/politcas-y-planes-en-salud/planes-en-salud/964-plan-nacional-de-salud-2016-2020/file
31. Ministerio de Salud. Análisis de la Situación de Salud 2018 [Internet]. San José, Costa Rica; 2019 [citado 1 de junio de 2020]. Disponible en: https://www.ministeriodesalud.go.cr/sobre_ministerio/memorias/memoria_2014_2018/memoria_institucional_2018.pdf

32. Peraza J, Vega A. Estudio de oferta de servicios odontológicos. Costa Rica 2012-2014. Rev Científica Odontológica. 2014;10(1): 15 .

33. Caja Costarricense de Seguro Social. Consolidado del Instrumento de Gestión de los Servicios de Odontología (IGEO)-2018. San José, Costa Rica: CCSS; 2019 p. 80. Report No.: DDSS-4445-19. 
